\title{
Teamwork training for mobile interdisciplinary teams
}

\author{
Prof. Dr. F. Bodendorf, Dr. R. Seitz \\ Information Systems II, University of Erlangen-Nuremberg \\ Lange Gasse 20, 90403 Nuremberg, German \\ Tel.: +49 $9115302-450$ (Fax: -379), \\ E-Mail:bodendorf@wiso.uni-erlangen.de
}

\section{Extended Abstract}

Successful management of business processes increasingly depends on flexibility and adaptability in the organization of work. In many cases you have to cope with ad-hoc-structured or ill-structured tasks. For virtual enterprises with frequently changing national or international partnerships this kind of processes will be the dominating one. More and more interdisciplinary or multicultural teams have to decide on their self-organization and self-management depending on the problem at hand. It is the university's task to train students in the management of teamwork as well as in modern techniques for cooperative and collaborative problem solving.

The training scenario is based on a team of three persons each playing a different role. Each of them contributes his know-how and skills acquired in different disciplines. The given task is to produce a compound document containing technical product information, cost data and marketing text. The product may be, for instance, a mountain bike (see Figure 1).

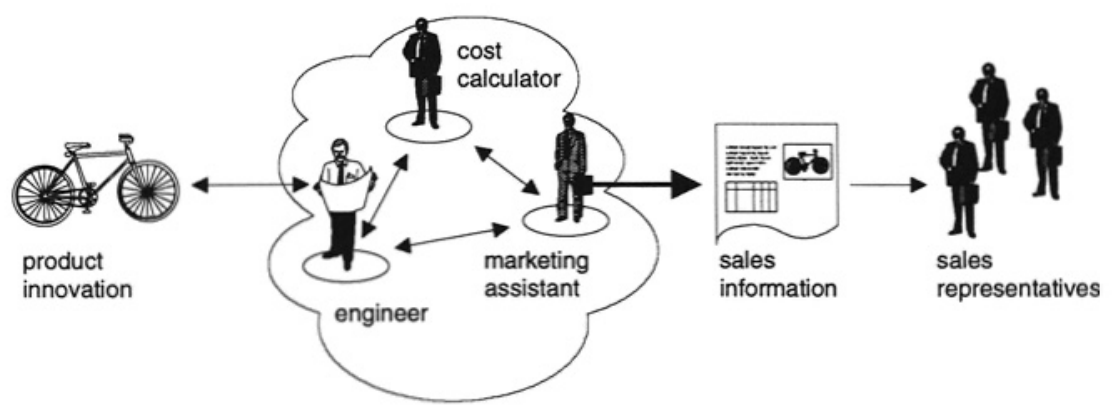

Figure 1 Teamwork Task.

The engineer is asked to improve the construction of a technical product. The cost calculator has to analysc the monetary effects of product variations. The marketing assistant adds information from a 
sales point of view. On the one hand each member of the team is responsible for a distinct part of the document. On the other hand all team members have to communicate and negotiate with one another in order to select the most appropriate product alternative and to harmonize the document. In this scenario students of different disciplines (engineering, cost accounting, marketing) have the opportunity to get a feeling for interdisciplinary collaboration. Using appropriate software and telecommunication equipment they conduct their teamwork in an asynchronous way independent of their location.

The teamwork process is shown in Figure 2. Subtasks assigned to the actors are outlined. Arrows represent trigger and information transfer functions.

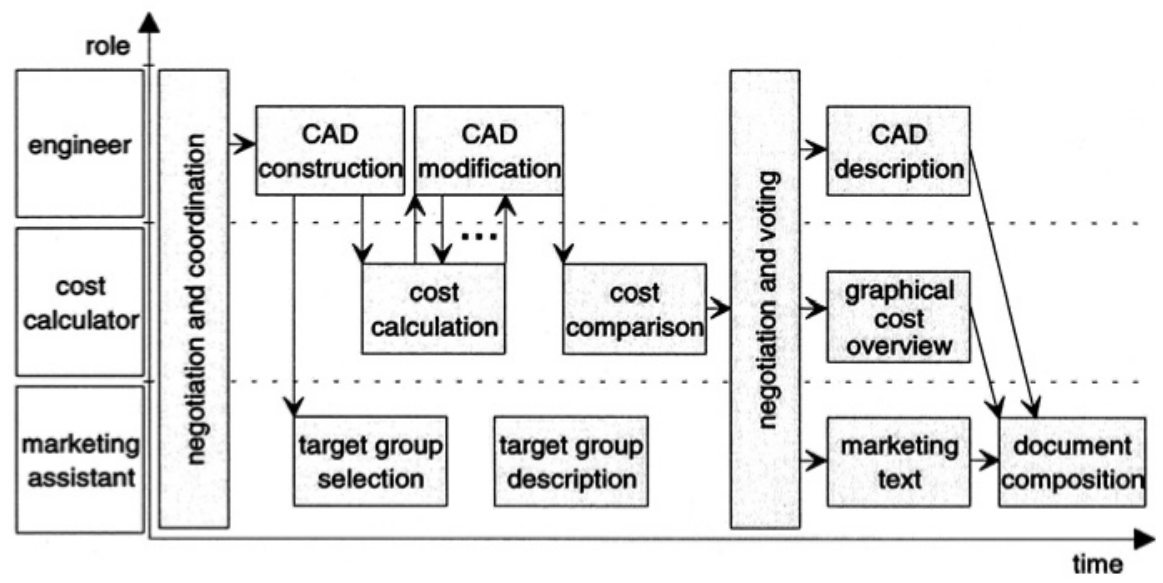

Figure 2 Teamwork Process.

The workgroup process begins with an electronic meeting involving all team members. They discuss distribution problems and decide to solve them by designing a new marketing initiative. Support is provided by an electronic meeting system offering assistance in the form discussion, brainstorming and voting facilities. The engineer begins to redesign parts of the mountain bike in accordance with the ideas and proposals worked out during the meeting. Evidently he uses a CAD system to achieve suitable results. When the engineer has completed his preliminary $\mathrm{CAD}$ sketch he informs the marketing assistant and contacts the cost calcuiator by sending him the construction file and the table of pieces. Using a spreadshect application the calculator now estimates the effects of redesign on production costs. At this point feedback cycles may occur. If the price is obviously too high redesign and cost catculation are repeated until the product costs reach a realistic level. Meanwhile the marketing assistant produces textual information for the selected target group. Should more than one alternative prove to be feasible the cost calculator produces a table comparing the redesigned concepts. To do this he consolidates several spreadsheets into a larger one. The consolidated spreadsheet is the subject of a new conference. Independent of their current location the members of the team join the electronic meeting, discuss the available options and vote on the most suitable alternative. In the final step the marketing assistant assembles all aspects of the document: drawing of the redesigned product, technical description, calculation spreadsheet, graphical cost display, text of marketing proposals. A software agent automatically records all events (time of task completion, acting persons). Thus the current state of the process can be monitored by each member at any time. 